\title{
Do psychiatrists sufficiently understand the statistical measures used in clinical research?
}

\author{
Kunwar A R*
}

Address: Kathmandu Medical College, Sinamangal, Kathmandu, Kathmandu, Nepal

Email*Correspondingauthor:arunkunwar@yahoo.com

ABSTRACT

Introduction: An understanding of the statistical concepts and epidemiological methods are essential for interpretation of any clinical trials. In this article, authors try to explore and find out, what the statistical understanding of psychiatrists by their own report and whether they feel confident in understanding some of the statistical concepts used in medical journals.

Material \& Method: This is a descriptive cross sectional study conducted among 33 fellow psychiatrists using an anonymous, self-reported questionnaire which inquired about their understanding of thirteen statistical concepts taken from a medical journal.

Results: Out of all the statistical measures inquired, the best understood were "standard deviation" $(97 \%)$ and "validity" (88\%); the least understood were "Kendall's Tau" $(6 \%)$ and "logit analysis" $(6 \%)$. Seventy six percent of those surveyed reported understanding the concept of "P-value" whereas only 36 percent understanding the "ANOVA test".

Conclusions: This study suggests that psychiatrists are not equipped with the statistical knowledge to critically evaluate primary research articles as reported in medical journals. Further larger studies should be conducted to improve our understanding of statistical knowledge among psychiatrists.

\section{Key words: Statistic, Psychiatrist, Medical Education, Evidence Based Medicine}

\section{INTRODUCTION}

An understanding of the statistical concepts and. epidemiological methods are essential for interpretation of any clinical trials. However, a number of studies conducted throughout the world have found that physicians are often not fully competent in basic research methods. In a study conducted among 289 family practitioners in Canada, it was found that only $50 \%$ of the respondents could critically appraise and interpret the results of medical articles. ${ }^{1}$ In yet another study, it was found that the most frequent errors committed by medical practitioners were in determination of the study type, sample size considerations, selection of the study population, proper use of parametric tests, analysis of repeated measures, descriptive statistics and determination of confidence intervals (CIs). ${ }^{2}$ The general statistical knowledge of the resident physicians of faculty of medicine in two universities of Turkey were also found to be insufficient. ${ }^{3}$ Statistics serves a major role in modern psychiatry and its understanding is of utmost importance to all psychiatrists. ${ }^{4}$ More often psychiatric researches resort to sophisticated research tools and techniques which also further reiterate the need to have basic statistical knowledge among psychiatrists. The basic objective of the study was to find out the whether or not psychiatrists understood a set of Kunwar. Do psychiatrists sufficiently understand the statistical......0014 statistical measures, drawn from medical journals. The survey was conducted to determine whether this lack of statistical comprehension is a widespread phenomenon in the psychiatric profession.

\section{MATERIAL AND METHOD}

This was a descriptive cross sectional study. It was conducted among 33 fellow psychiatrists at the Department of Psychiatry at SUNY-Upstate Medical University, Syracuse, New York and surrounding community from October 29 to November 22003 using an anonymous, self-reported questionnaire. The statistical measures incorporated in the questionnaire were

- Regression analysis . Standard deviation

- T-Test - Chi-square

- Kendall's Tau - Two-tailed T-test

- Validity

- Odds Ratio

- Logit analysis

- ANOVA test 
It probed their knowledge of statistical techniques and their difficulties in interpreting them in medical journals. A verbal informed consent was obtained from the respondents. Only medical practitioners who had specialized in psychiatry were selected for the study. Ethical approval was obtained from institution review board.

Data was entered and analysis was done in SPSS (Version 11.5, SPSS Inc., and Chicago, USA)

\section{RESULTS}

Table 1 show that respondents' understanding of these concepts varies considerably. For example, 76 percent of those surveyed understood the concept of "P-value" whereas only 36 percent understood the "ANOVA test". Among this group of statistical measures, the best understood were "standard deviation" and "validity"; the least understood were "Kendall's Tau" and "logit analysis". Although logit analysis is widely used in many clinical researches for risk assessment ${ }^{6}$ however, only two of the respondents had knowledge about this statistical method. Table 1: Do you have the knowledge of statistical techniques mentioned below and do you feel confident in interpreting them in medical journals?

\begin{tabular}{|lll|}
\hline Statistical measures & Yes (\%) & No (\%) \\
\hline Regression analysis & $15(45)$ & $18(55)$ \\
\hline Standard deviation & $32(97)$ & $1(3)$ \\
\hline T-Test & $24(73)$ & $9(27)$ \\
\hline Chi-square & $24(73)$ & $9(9)$ \\
\hline Kendall's Tau & $2(6)$ & $31(94)$ \\
\hline Two-tailed T-test & $16(48)$ & $17(52)$ \\
\hline Validity & $29(88)$ & $4(12)$ \\
\hline Relative risk & $26(79)$ & $7(31)$ \\
\hline Odds Ratio & $23(70)$ & $10(30)$ \\
\hline P-value & $25(76)$ & $8(24)$ \\
\hline Logit analysis & $2(6)$ & $31(94)$ \\
\hline Person's coefficient & $6(18)$ & $27(82)$ \\
\hline ANOVA test & $12(36)$ & $21(64)$ \\
\hline
\end{tabular}

\section{DISCUSSION}

In this day and age when medical science is evolving rapidly, it is important to keep up to date with new advances in our field. Most of the practicing physicians will do this by reading medical journals. Having a good understanding of the statistics is essential for interpretation of clinical research published in these journals. This study has tried to explore whether psychiatrist have sufficient statistical understanding sothat they can make appropriate interpretation of published articles. We found that psychiatrist did not have sufficient understanding of many statistical concepts. Our finding is comparable to earlier published findings from similar studies of physicians in other medical specialties that also found a gap in statistical comprehension. ${ }^{2.3}$

This study has limitations (small sample size and was limited geographical area) but above finding is no surprise, as this study was initiated by authors as they often have encountered difficulty in interpreting various statistical results reported in medical journals) due to a lack of sufficient statistical knowledge. The authors wanted to determine whether this lack of statistical comprehension is a widespread phenomenon in the psychiatric profession and this study has suggested that's probably true. As mentioned above, lack of good statistical knowledge is a barrier to understanding and interpreting clinical trials. So, we all should take this seriously.

\section{CONCLUSION}

This study suggests that psychiatrists are not equipped with the statistical knowledge to critically evaluate primary research articles as reported in medical journals. Further larger studies should be conducted to improve our understanding of statistical knowledge among psychiatrists and also to explore possible remedial approaches such as increasing the teaching of statistics in medical schools and residency programs and the inclusion of statistical tutorials in medical journals which all could bridge this gap.

\section{REFERENCES}

1. Godwin M, Seguin R. Critical appraisal skills of family physicians in Ontario, Canada. BMC Med Educ. 2003 Dec; 3:10.

2. Novack L, Jotkowitz A, Knyazer B, et al. Evidencebased medicine: assessment of knowledge of basic epidemiological and research methods among medical doctors. Postgrad Med J. 2006; 82: 817-822.

3. Ercan I, Ozkaya G, Ocakoglu G, et al. Determining Biostatistics Knowledge of Students and Physicians in Medical School. 1Biostatistics Department, Faculty of Medicine, Uludag University, Bursa, Turkey; 2007 (Unpublished).

4. Everitt BS. Statistics in Psychiatry. Statistical science. 1987;2(2):107-134.

5. The role of statistics in psychiatry. Psychological Medicine. 1985; 15: 471-476.

6. Daniel WW. Biostatistics. Delhi: Wiley India (P) Ltd.; 2009. 